\title{
Protostellar collapse: radiative and magnetic feedbacks on small-scale fragmentation ${ }^{\star}$
}

\author{
B. Commerçon ${ }^{1,2,3}$, P. Hennebelle ${ }^{4}$, E. Audit ${ }^{3}$, G. Chabrier ${ }^{2}$, and R. Teyssier ${ }^{3}$ \\ 1 Max Planck Institute for Astronomy, Königstuhl 17, 69117 Heidelberg, Germany \\ e-mail: benoit@mpia-hd.mpg.de \\ 2 École Normale Supérieure de Lyon, CRAL, UMR 5574 CNRS, Université de Lyon, 46 allée d'Italie, 69364 Lyon Cedex 07, France \\ 3 Laboratoire AIM, CEA/DSM - CNRS - Université Paris Diderot, IRFU/SAp, 91191 Gif sur Yvette, France \\ ${ }^{4}$ Laboratoire de radioastronomie, UMR 8112 CNRS, École Normale Supérieure et Observatoire de Paris, 24 rue Lhomond, \\ 75231 Paris Cedex 05, France
}

Received 3 November 2009 / Accepted 12 January 2010

\section{ABSTRACT}

\begin{abstract}
Context. Both radiative transfer and magnetic field are understood to have strong impacts on the collapse and the fragmentation of prestellar dense cores, but no consistent calculation exists on these scales.

Aims. We perform the first radiation-magneto-hydrodynamics numerical calculations on a prestellar core scale.

Methods. We present original AMR calculations including that of a magnetic field (in the ideal MHD limit) and radiative transfer, within the flux-limited diffusion approximation, of the collapse of a $1 M_{\odot}$ dense core. We compare the results with calculations performed with a barotropic EOS.

Results. We show that radiative transfer has an important impact on the collapse and the fragmentation, by means of the cooling or heating of the gas, and its importance depends on the magnetic field. A stronger field yields a more significant magnetic braking, increasing the accretion rate and thus the effect of the radiative feedback. Even for a strongly magnetized core, where the dynamics of the collapse is dominated by the magnetic field, radiative transfer is crucial to determine the temperature and optical depth distributions, two potentially accessible observational diagnostics. A barotropic EOS cannot account for realistic fragmentation. The diffusivity of the numerical scheme, however, is found to strongly affect the output of the collapse, leading eventually to spurious fragmentation.

Conclusions. Both radiative transfer and magnetic field must be included in numerical calculations of star formation to obtain realistic collapse configurations and observable signatures. Nevertheless, the numerical resolution and the robustness of the solver are of prime importance to obtain reliable results. When using an accurate solver, the fragmentation is found to always remain inhibited by the magnetic field, at least in the ideal MHD limit, even when radiative transfer is included.
\end{abstract}

Key words. magnetohydrodynamics (MHD) - radiative transfer - stars: formation - stars: low mass - ISM: clouds ISM: kinematics and dynamics

\section{Introduction}

Understanding star formation is one of the most challenging problems in contemporary astrophysics and numerical calculations provide a useful approach to its investigation. Because of steadily improv computer performances, numerical calculations can simulate more and more physical processes of which the coupling between matter and radiation is a major issue. Until the formation of the first Larson core (Larson 1969), the accreting gas can freely radiate into space and is nearly isothermal (optically thin regime). Once the gas becomes dense enough $\left(\rho>10^{-13} \mathrm{~g} \mathrm{~cm}^{-3}\right)$, the radiation is trapped and the gas begins to heat up (optically thick regime). The transition between these two regimes controls the collapse and fragmentation of the cloud. The cooler the gas, the more important the fragmentation. Another key issue in star formation is the role of the magnetic field, as dense cores are observed to exhibit coherent magnetic structures (e.g., Heiles \& Crutcher 2005).
Several authors have investigated the impact of both radiation (Boss et al. 2000; Whitehouse \& Bate 2006; Attwood et al. 2009; Krumholz et al. 2007; Bate 2009; Offner et al. 2009) and magnetic field (e.g., Mellon \& Li 2009; Banerjee \& Pudritz 2006; Hennebelle \& Teyssier 2008; Machida et al. 2008; Price \& Bate 2007, 2008) on the star-formation process. However, only Price \& Bate (2009) have to date performed calculations including both magnetic field and radiative feedback, using a SPH method, although their calculations explore relatively large scales. In this Letter, we present the first full radiation-magnetohydrodynamics (RMHD) calculations of the protostellar collapse on small scales. We investigate in detail the impact of both magnetic field (in the limit of ideal MHD) and radiative feedback on the fragmentation process and the launching of outflows. The results are compared with those obtained with a barotropic equation of state (EOS), which crudely mimics the transition from the isothermal to adiabatic regime, to assess the importance of a proper treatment of radiative transfer on the collapse.

$\star$ Appendices A and B are only available in electronic form at http://www . aanda. org 

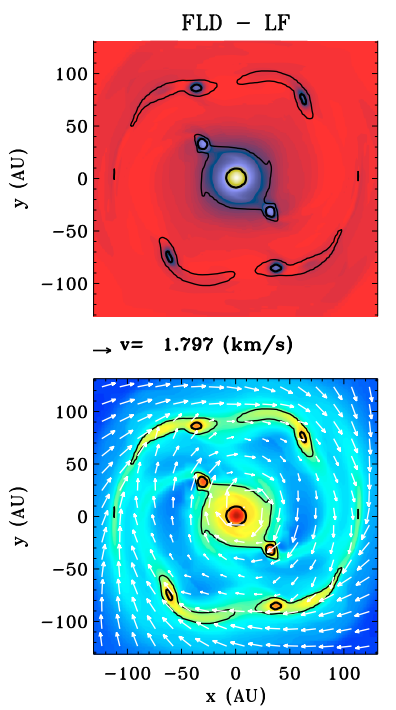

FLD - HLLD

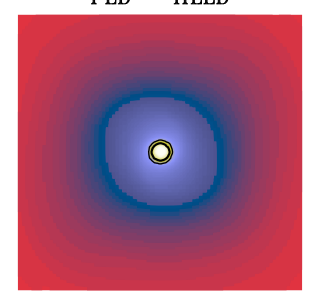

$\rightarrow \mathrm{v}=2.121(\mathrm{~km} / \mathrm{s})$

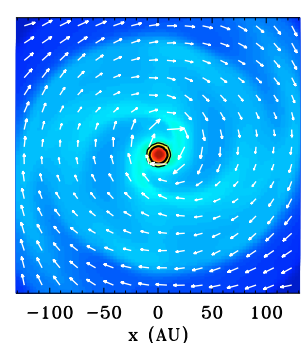

Barotrop - LF

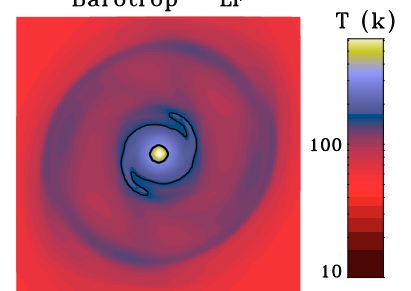

$\rightarrow \mathrm{v}=1.595(\mathrm{~km} / \mathrm{s})$

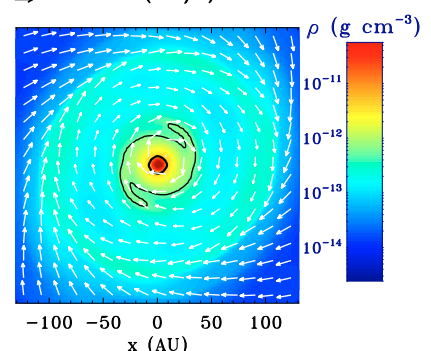

Fig. 1. Case $\mu=20$ : density (bottom) and temperature (top) maps in the equatorial plane at time $t=38.6$ kyr: FLD approximation with LF (left), FLD with HLLD (middle), and barotropic EOS with LF (right).

\section{Numerical method and initial conditions}

We use the RAMSES code (Teyssier 2002) based on a Eulerian formalism (grid based method with adaptive mesh refinement). We solve the transfer equations in the flux-limited diffusion (FLD, Minerbo 1978) in the comoving frame (fluid frame) to evaluate the radiative quantities. The ideal MHD equations are integrated using an unsplit second-order Godunov scheme (Fromang et al. 2006). Coupling terms between matter and radiation, as well as radiation transport, are integrated implicitly to handle the very short heating, cooling, and diffusion timescales. Details of the numerical method and its implementation will be provided in a forthcoming paper.

Calculations were performed using either the rather diffusive Lax Friedrich (LF) Riemann solver or the more accurate HLLD Riemann solver (Miyoshi \& Kusano 2005). Following up on former studies (Commerçon et al. 2008), we impose at least 10 cells per Jeans length as a grid refinement criterion (parameter $N_{\mathrm{J}}$ ). The initial resolution of the grid contains $64^{3}$ cells. We use the low temperature opacities of Semenov et al. (2003), parametrized as functions of the gas temperature and density. In contrast, the non-radiative calculations were performed with a barotropic EOS $P / \rho=c_{\mathrm{s} 0}^{2}\left[1+\left(\rho / \rho_{\mathrm{ad}}\right)^{\gamma-1}\right]$, where $\rho_{\text {ad }}=2.3 \times 10^{-13} \mathrm{~g} \mathrm{~cm}^{-3}$ is the critical density at which the gas becomes adiabatic, $\gamma$ (set to be 5/3) is the adiabatic index, and $c_{\mathrm{s} 0}$ is the isothermal sound speed.

We adopt initial conditions, similar to those chosen in previous studies (e.g. Commerçon et al. 2008). We consider a uniform-density sphere of molecular gas, rotating about the $z$ axis with a uniform angular velocity. In the present study, the prestellar core mass is fixed at $M_{0}=1 M_{\odot}$ and the temperature at $11 \mathrm{~K}$, which corresponds to $c_{\mathrm{s} 0} \sim 0.19 \mathrm{~km} \mathrm{~s}^{-1}$. To promote fragmentation, we use an $\mathrm{m}=2$ azimuthal density perturbation with an amplitude of $10 \%$. The magnetic field is initially uniform and parallel to the rotation axis. The strength of the magnetic field is expressed in terms of the mass-to-flux to critical mass-to-flux ratio $\mu=\left(M_{0} / \Phi\right) /\left(M_{0} / \Phi\right)_{c}$. The initial energy balance is determined by two dimensionless parameters, namely the ratio of the thermal to gravitational energies $\alpha=$ 0.37 , and rotational over gravitational energies $\beta=0.045$. The corresponding free-fall time and orbital time are, respectively, $t_{\mathrm{ff}} \sim 33 \mathrm{kyr}$ and $t_{\mathrm{orb}} \sim 1.85 \times 10^{2} \mathrm{kyr}$. The initial density is $\rho_{0}=3.97 \times 10^{-18} \mathrm{~g} \mathrm{~cm}^{-3}$.

\section{Results}

\subsection{Case $\mu=20$}

We first present the results for $\mu=20$, i.e., a weakly magnetized core. We compare FLD calculations performed with the two Riemann solvers (LF or HLLD) with calculations performed with the barotropic EOS and the LF solver. The grid refinement criterion is $N_{\mathrm{J}}=15$. Performing similar barotropic calculations, Hennebelle \& Teyssier (2008) do not report any fragmentation.

Figure 1 portrays temperature (top) and density (bottom) maps in the equatorial plane for the three aforementioned calculations at the same time $t \sim 38 \mathrm{kyr}$. The black contours represent the transition between the optically thin and thick regions (thin contour) and the quasi-adiabatic regions. The FLD case with the LF solver yields a multiple fragmentation, a central fragment of mass $5.2 \times 10^{-2} M_{\odot}$ and several (depending on the time and resolution) orbiting fragments of mass $\sim 2-4 \times 10^{-3} M_{\odot}$ having separations ranging from $\sim 40 \mathrm{AU}$ to $\sim 100 \mathrm{AU}$, whereas no fragmentation occurs in the two other cases. The orbiting fragments in the FLD-LF simulation are quite warm $(\sim 40 \mathrm{~K})$, while the disc is cold $(\sim 11 \mathrm{~K})$. In all simulations, the central fragment $(T>500 \mathrm{~K})$ corresponds to an adiabatic region, where the gas cannot radiate away its compressional energy. In the barotropic case, the mass of the central fragment is $4.9 \times 10^{-2} M_{\odot}$. The outer parts of the disc formed are much denser and warmer than for the two other cases, even though the gas is optically thin and should cool efficiently, as illustrated in the FLD-LF calculations. The corresponding values of the Toomre parameter and Jeans length are greater than in the FLD-LF case. This heating, however, is spurious and reflects the approximate treatment of radiative cooling with a barotropic approximation, where temperature is determined by the density.

The most interesting case is the FLD case with the HLLD Riemann solver. No fragmentation occurs in that case. The matter falls onto a central core of mass $\sim 7.3 \times 10^{-2} M_{\odot}$. With this less diffusive solver, the generation of a toroidal magnetic field is more efficient. The interplay of magnetic field lines and velocity gradients leads to the effective expansion of a magnetic "tower" in the vertical direction (Hennebelle \& Fromang 2008). The first 
B. Commerçon et al.: Protostellar collapse: radiative and magnetic feedbacks on small-scale fragmentation
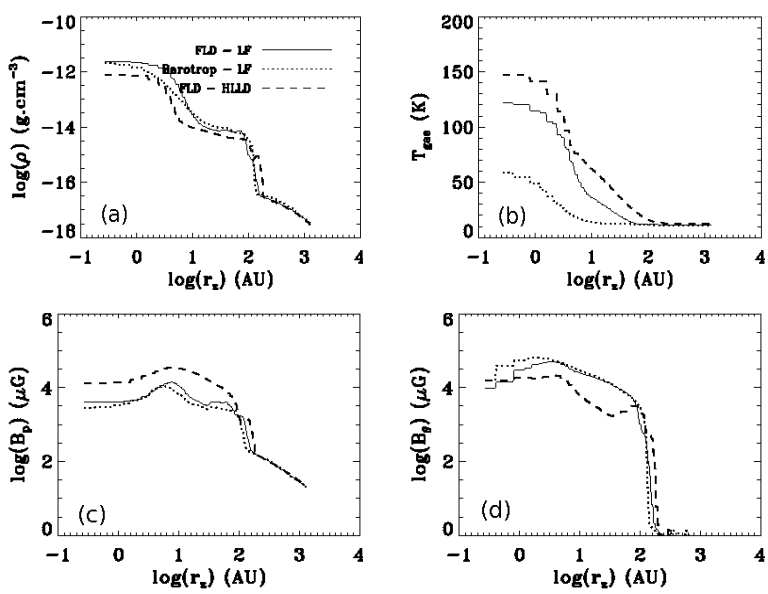

Fig. 2. Case $\mu=20$ : profiles of density a), temperature b), poloidal c), and toroidal d) magnetic field components as a function of height above the equatorial plane at a cylindrical radius of $10 \mathrm{AU}$, for the same calculations and at the same time as in Fig. 1.

consequence of this more robust (less diffusive) solver is that the disc formed is less massive in the equatorial plane than with the LF solver.

Figure 2 shows the density, temperature, and both poloidal and toroidal magnetic field components profiles as a function of height above the equatorial plane at a cylindrical radius of $10 \mathrm{AU}$, for the three aforementioned calculations, at the same time. We note that the extent of the magnetic tower depends on (increases with) resolution. The magnetic tower is less dense close to the equatorial plane, since the gas is spread in the magnetic bubble. In contrast to the barotropic case, where the temperature falls to $10 \mathrm{~K}$ at a distance $>10 \mathrm{AU}$, the radiative feedback extends vertically up to $\sim 100$ AU with radiative transfer (FLD). Although toroidal magnetic field generation is more effective with HLLD than with LF, the toroidal magnetic field in the FLD-LF case is stronger at small radii. This is because of the denser disc formed, which compresses and twists the magnetic lines in the region close to the equatorial plane. At a distance of $100 \mathrm{AU}$, the toroidal magnetic field component in the vertical direction is one order of magnitude greater with HLLD than with LF. This favours the extension of the magnetic tower. The poloidal magnetic field component is stronger everywhere in the HLLD case, since matter is less compressed.

Figure 3 displays the profiles of the magnetic braking force at $10 \mathrm{AU}$ above the equatorial plane and as a function of height above the equatorial plane at a cylindrical radius of $10 \mathrm{AU}$, for the three same calculations. In the equatorial plane, the magnetic braking obtained with the LF solver or in the barotropic case is barely significant. Magnetic braking favours faster accretion to the central object and thus leads to a larger amount of kinetic energy being radiated away at the first core accretion shock (all the infalling gas kinetic energy is radiated away at the first Larson core accretion shock). The gas is then heated significantly around the central core. In contrast, there is more angular momentum in the (diffusive) FLD-LF case, which promotes fragmentation at the centrifugal barrier. The amount of accretion onto the central fragment is thus lower and the heating caused by accretion is less important. Magnetic braking force as a function of height above the plane is similar for the three calculations. We thus identify two important processes, both quantitatively affected by the numerical treatment: the presence of a magnetic tower, whose extension depends on the numerical resolution, and
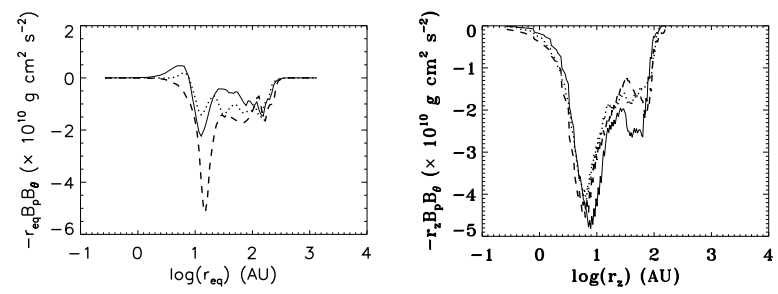

Fig. 3. Case $\mu=20$ : profiles of magnetic braking force at $10 \mathrm{AU}$ above the equatorial plane (left) and as a function of height above the equatorial plane at a cylindrical radius of $10 \mathrm{AU}$ (right), for the same calculations (same legend) and at the same time as in Fig. 1.

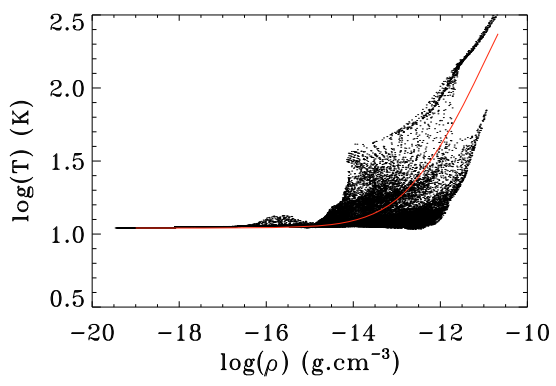

Fig. 4. Case $\mu=20$ : temperature-density plot for each cell in the FLDLF calculations (black) and with the barotropic EOS (red line).

strong magnetic braking in the equatorial plane, whose strength depends on the diffusivity of the solver.

Figure 4 shows the temperature - density distribution for the FLD calculations with the LF solver. The isothermal and adiabatic regimes are recovered at low and high density $(\rho<$ $10^{-16} \mathrm{~g} \mathrm{~cm}^{-3}$ and $\left.\rho>10^{-12.5} \mathrm{~g} \mathrm{~cm}^{-3}\right)$. In-between, we observe a dispersion in the $(T, \rho)$ plane, where matter can be hotter at low density and cooler at high density compared with the barotropic treatment (red solid line). As seen in the figure, the fragments eventually reach different isentropes. The central fragment lies on the highest entropy level, while the orbiting fragments end up on cooler isentropes. The barotropic EOS cannot reproduce this spread in temperature at a given density. The entropy and the minimum Jeans mass in that case are determined by the choice of $\rho_{\text {ad }}$. In the FLD-LF case, cooling is more efficient; material in the equatorial plane cools by radiating in the vertical directions, where the gas is optically thin. However, this result is mainly affected by the diffusivity of the Riemann solver. In the FLDHLLD case, all the material was heated by the central fragment accretion luminosity and distributed above the barotropic adiabat. In the FLD-LF case, the fragmentation is the combined result of the inefficient magnetic braking and the efficient cooling caused by the FLD. This illustrates the importance and complexity of the interplay between the magnetic field and radiative transfer.

\subsection{Case $\mu=5$}

We now consider a strongly magnetized core, with $\mu=5$, and explore the impact of radiative transfer on both the outflow and the temperature distribution in that case. Calculations were performed with the HLLD solver and $N_{\mathrm{J}}=10$. For this more magnetized model, the magnetic field lines determine the dynamics of the collapse and, in contrast to the previous case, it is the flow that adjusts to the magnetic field topology rather than the opposite.

Figure 5 compares temperature and density maps in the $y z$-plane for the FLD and barotropic calculations, at $t=1.14 t_{\mathrm{ff}} \sim$ 38 kyr. In both cases, an outflow is launched, with similar 

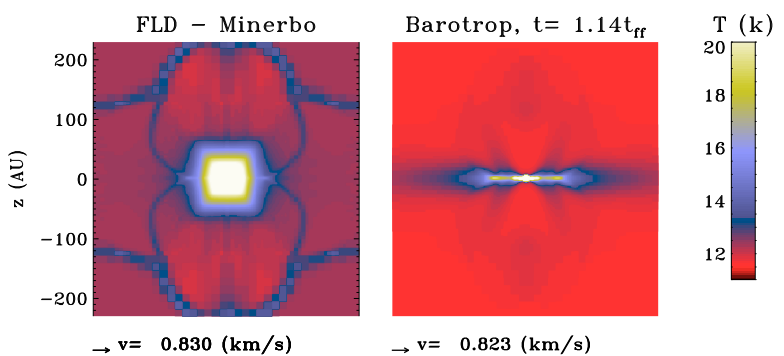

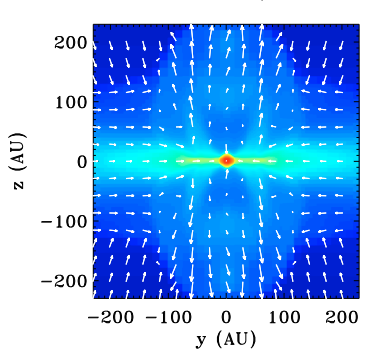

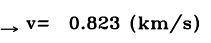

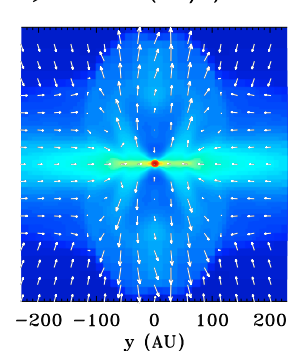

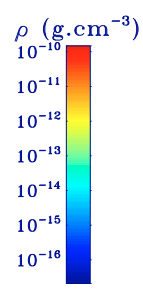

Fig. 5. Case $\mu=5$ : temperature (top) and density (bottom) maps in the $x y$-plane for FLD and barotropic EOS calculations (right).

propagation and opening angles. The density patterns are also similar. The temperature distributions, however, differ drastically. As shown in Hennebelle \& Fromang (2008), a pseudowarm disc is formed in the equatorial plane in the barotropic case. We also note the lack of significant heating in the vertical direction with the barotropic EOS. Since the density within the outflow is low, $\rho \sim 5 \times 10^{-15} \mathrm{~g} \mathrm{~cm}^{-3}$, this implies a low temperature, which quickly falls to $11 \mathrm{~K} 10 \mathrm{AU}$ from the center. When including a more proper treatment of radiation, with the FLD, the radiation escapes preferentially in the vertical direction (along the $z$-axis) and heats the gas out to about 100 AU. The highest outflow velocity, $v_{\mathrm{r}} \sim 1.4 \mathrm{~km} \mathrm{~s}^{-1}$, is obtained in the barotropic case, while $v_{\mathrm{r}} \sim 1.1 \mathrm{~km} \mathrm{~s}^{-1}$ in the FLD calculation. The border line between the outflow and the infalling gas shows a small spike, where the material is shocked and heated. The toroidal magnetic field component profiles are in good agreement with the results of Hennebelle \& Fromang (2008) and exhibit a nearly constant plateau in the region of the outflow. For our present study of a $1 M_{\odot}$ core, radiative cooling in the $\mu=5$ case is found to be almost inconsequential to the collapse. The cores are never found to fragment. For more massive cores with stronger radiative feedback, however, the heating in the outflow is likely to be more dynamically significant (Krumholz et al. 2007).

\section{Summary and discussion}

We have explored the effects of both radiative transfer and magnetic fields (in the limit of ideal MHD) on the fragmentation of a $1 M_{\odot}$ prestellar core. In agreement with previous non-magnetic studies by, for instance, Boss et al. (2000), Whitehouse \& Bate (2006), and Krumholz et al. (2007), we show that a proper treatment of radiation is important to correctly describe this process. Radiative transfer enables the gas to either cool significantly or heat in different regions of equal densities, whereas a barotropic EOS approximation implies that the cooling and the heating are fixed by the density.

In the case $\mu=20$, where both the behaviour of the flow and the magnetic field affect the dynamics of the collapse, we show that radiative transfer has an important impact on the final structure. A barotropic approximation can account for neither the cooling of the dense and rotating gas in the equatorial plane nor the heating of the less dense gas in the vertical direction, where radiation is found to escape preferentially. Although a value $\mu=20$ is inconsistent with observations, this case clearly illustrates the impact of a proper treatment of radiative transfer on the collapse and fragmentation of prestellar cores. For the strongly magnetized $\mu=5$ case, the dynamics of the collapse is dominated by the magnetic field. In that case, a proper treatment of radiative cooling is less consequential to the collapse itself but is crucial for deriving correct optical depth and temperature distributions, two accessible observational diagnostics. The temperature distribution with the FLD is indeed found to depend strongly on the geometry, whereas the barotropic approximation yields a nearly uniform distribution. We also note that in the $\mu=5$ case, we always obtain an outflow, even when using the more diffusive LF Riemann solver. These outflows cannot be obtained using SPH calculations. We also performed FLD test calculations including the radiative feedback from a pseudo central newborn star and found that, for our $1 M_{\odot}$ core case, it is inconsequential on the length and time scales of interest.

The spurious diffusivity caused by either the numerical resolution or the hydrodynamic solver, however, is found to significantly affect the collapse and fragmentation. A less diffusive numerical scheme (HLLD instead of LF) produces strong magnetic braking, which transports angular momentum. Material then falls onto the central core, leading to a higher accretion luminosity. A high numerical resolution is also crucial in the vertical direction to accurately describe the growth of the magnetic tower, which spreads the gas around the central object.

Acknowledgements. We thank the anonymous referee for comments which have improved the paper significantly. Calculations have been performed at CEA on the DAPHPC cluster. We acknowledge funding from the European Community via the P7/2007-2013 Grant Agreement no. 247060.

\section{References}

Attwood, R. E., Goodwin, S. P., Stamatellos, D., \& Whitworth, A. P. 2009, A\&A, 495, 201

Banerjee, R., \& Pudritz, R. E. 2006, ApJ, 641, 949

Bate, M. R. 2009, MNRAS, 392, 1363

Boss, A. P., Fisher, R. T., Klein, R. I., \& McKee, C. F. 2000, ApJ, 528, 325

Commerçon, B., Hennebelle, P., Audit, E., Chabrier, G., \& Teyssier, R. 2008, A\&A, 482, 371

Fromang, S., Hennebelle, P., \& Teyssier, R. 2006, A\&A, 457, 371

Heiles, C., \& Crutcher, R. 2005, in Cosmic Magnetic Fields, LNP, 664, 137

Hennebelle, P., \& Fromang, S. 2008, A\&A, 477, 9

Hennebelle, P., \& Teyssier, R. 2008, A\&A, 477, 25

Krumholz, M. R., Klein, R. I., \& McKee, C. F. 2007, ApJ, 656, 959

Larson, R. B. 1969, MNRAS, 145, 271

Machida, M. N., Tomisaka, K., Matsumoto, T., \& Inutsuka, S.-i. 2008, ApJ, 677, 327

Mellon, R. R., \& Li, Z.-Y. 2009, ApJ, 698, 922

Minerbo, G. N. 1978, J. Quant. Spec. Radiat. Transf., 20, 541

Miyoshi, T., \& Kusano, K. 2005, J. Comp. Phys., 208, 315

Offner, S. S. R., Klein, R. I., McKee, C. F., \& Krumholz, M. R. 2009, ApJ, 703, 131

Price, D. J., \& Bate, M. R. 2007, MNRAS, 377, 77

Price, D. J., \& Bate, M. R. 2008, MNRAS, 385, 1820

Price, D. J., \& Bate, M. R. 2009, MNRAS, 398, 33

Semenov, D., Henning, T., Helling, C., Ilgner, M., \& Sedlmayr, E. 2003, A\&A, 410, 611

Teyssier, R. 2002, A\&A, 385, 337

Whitehouse, S. C., \& Bate, M. R. 2006, MNRAS, 367, 32

Pages 5 to 6 are available in the electronic edition of the journal at http: //www . aanda. org 


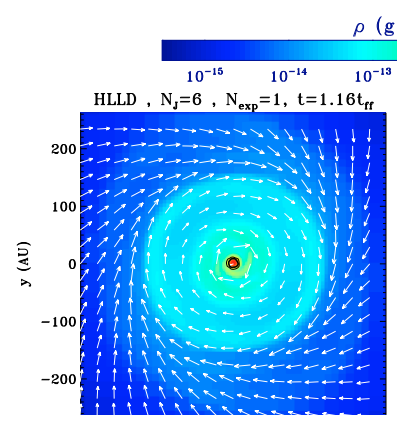

$L F, N_{\mathrm{J}}=20, \mathrm{~N}_{\mathrm{exp}}=4$

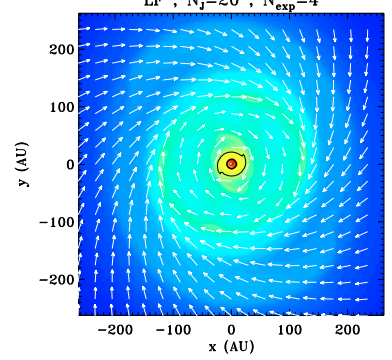

$\rho\left(\mathrm{g} \cdot \mathrm{cm}^{-3}\right)$

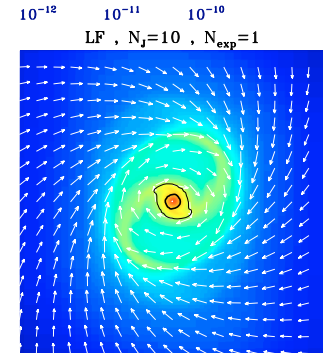

$\mathrm{LF}, \mathrm{N}_{\mathrm{s}}=6, \mathrm{~N}_{\mathrm{ev}}=1$

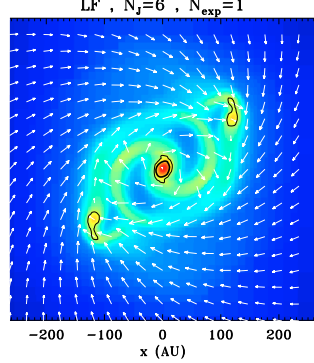

Fig. A.1. Case $\mu=20$ : density maps in the $x y$-plane at time $t \sim 1.16 t_{\mathrm{ff}}$ for 4 barotropic EOS calculations with HLLD, $N_{\mathrm{J}}=6$ and $N_{\exp }=1$; LF, $N_{\mathrm{J}}=20$ and $N_{\text {exp }}=4 ; \mathrm{LF}, N_{\mathrm{J}}=10$ and $N_{\text {exp }}=1 ; \mathrm{LF}, N_{\mathrm{J}}=6$ and $N_{\exp }=1$.

\section{Appendix A: Note on the influence of both the Riemann solver and the numerical resolution}

We present a short convergence study to investigate the effect of both the Riemann solver and the numerical resolution on the fragmentation process. We show in Sect. 3.1 that the use of the LF Riemann solver leads to inaccurate results. We perform calculations using the barotropic EOS and the HLLD or LF solvers at various numerical resolutions. The latter is determined by 2 parameters: $N_{\mathrm{J}}$, the number of points per Jeans length, and $N_{\text {exp }}$, which indicates the extent of the mesh around a refined cell (if a cell is flagged for refinement, then the $N_{\text {exp }}$ cells around the flagged cell, in each direction, will also be refined). A large $N_{\text {exp }}$ provides a smoother transition between the levels of the AMR grid. All calculations presented in Sect. 3.1 have been run with $N_{\exp }=4$. We report results of 4 calculations, performed for: HLLD, $N_{\mathrm{J}}=6$ and $N_{\exp }=1 ; \mathrm{LF}, N_{\mathrm{J}}=20$ and $N_{\exp }=4 ; \mathrm{LF}$, $N_{\mathrm{J}}=10$ and $N_{\text {exp }}=1$; and LF, $N_{\mathrm{J}}=6$ and $N_{\exp }=1$.

Figure A.1 portrays the density maps in the equatorial plane for the 4 calculations, at time $t \sim 1.16 t_{\mathrm{ff}}$. The calculations performed with the LF solver, $N_{\mathrm{J}}=6$ and $N_{\exp }=1$ clearly diverge from the others, since it fragments. The lack of resolution clearly induces inaccurate fragmentation. The three other calculations are qualitatively similar (no fragmentation) and agree with Hennebelle \& Teyssier (2008) results (obtained using a Roe-type Riemann solver). This indicates that the numerical resolution should be enhanced with the LF solver to avoid spurious effects caused by the diffusivity of the solver. We note that these differences are more important in the case of FLD calculations, since depending on the strength of the magnetic braking, radiative transfer can have two opposite effects. If magnetic braking is insignificant (with poor resolution and a diffuse Riemann solver), the material at the centrifugal barrier cools and then fragments. On the other hand, when the magnetic braking is efficient (with HLLD or a high resolution), the infall velocity and consequently, the accretion luminosity, become higher, which prevents fragmentation occurring (as material loses angular momentum and heats up).

\section{Appendix B: Case $\mu=1000$}

For this quasi-hydro case, the initial dense core is highly gravitationally unstable. We performed three types of calculations with the LF Riemann solver: one with the barotropic EOS and $\gamma=5 / 3$, and two with the FLD and different adiabatic exponents $(\gamma=5 / 3$ and $\gamma=7 / 5)$. The value $\gamma=7 / 5$ is more appropriate for $T>100 \mathrm{~K}$, when the rotational degrees of freedom of $\mathrm{H}_{2}$ are excited (e.g., Machida et al. 2008). Using $\gamma=7 / 5$ yields cooler adiabatic cores than with $\gamma=5 / 3$ at the same density. Higher densities and temperatures in the core are thus reached more rapidly.

Figure A.2 portrays density and temperature maps in the equatorial plane for the three aforementioned calculations at a time $t=1.15 t_{\mathrm{ff}}$. Temperature maps range from $11.4 \mathrm{~K}$ to $20 \mathrm{~K}$. As pointed out in Commerçon et al. (2008), the horizon of predictability is very short when the initial thermal support is low. Calculations do not show a convergence to the same fragmentation pattern, since we do not integrate the same system equations. The FLD calculations yield different fragmentation modes for the two values of $\gamma$ 's: for $\gamma=7 / 5$, we obtain a central object and two satellites (4 at later times), whereas for $\gamma=5 / 3$, we obtain a ring fragmentation pattern with orbiting fragments linked by a bar. The barotropic EOS calculation does not produce a central object. From the temperature map of the FLD case with $\gamma=7 / 5$, we see that the central region is quite hot. Fragments are compressed more and radiate more energy than for $\gamma=5 / 3$. Fragments also form more rapidly than the time required for the radiative feedback to become significant (at beginning of the second collapse). 
A\&A 510, L3 (2010)

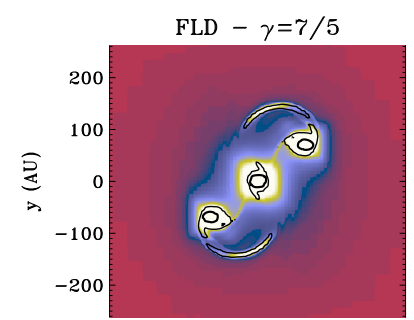

$\rightarrow \mathrm{v}=2.327(\mathrm{~km} / \mathrm{s})$

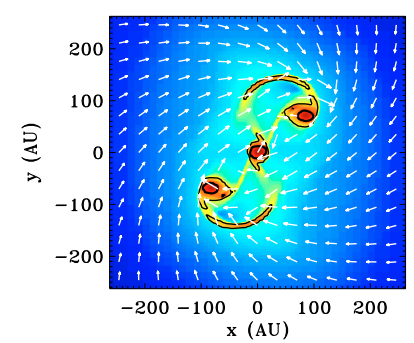

FLD $-\gamma=5 / 3$

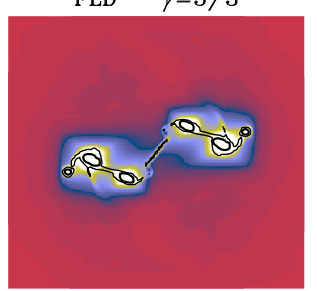

$\rightarrow \mathrm{v}=2.594(\mathrm{~km} / \mathrm{s})$

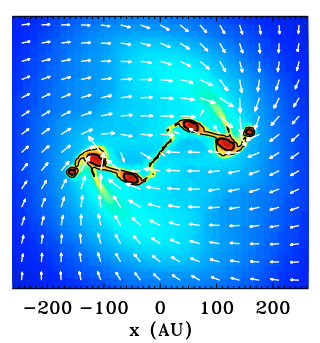

Barotrop, $\rho_{\mathrm{c}}=2.3 \times 10^{-13} \mathrm{~g} \mathrm{~cm}^{-3}$

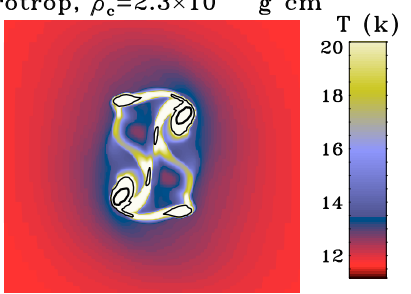

$\rightarrow \mathrm{v}=2.489(\mathrm{~km} / \mathrm{s})$

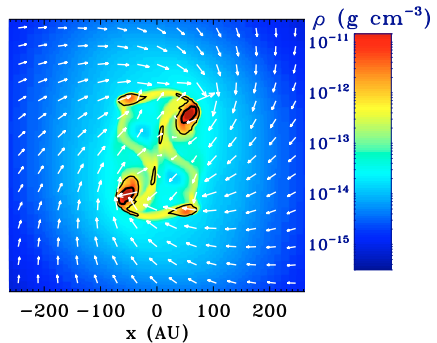

Fig. A.2. Density and temperature maps in the equatorial plane at time $t=38.3 \mathrm{kyr}\left(1.15 t_{\mathrm{ff}}\right)$, for the 3 unmagnetized cases: FLD approximation and $\gamma=7 / 5$ (left), the FLD approximation and $\gamma=5 / 3$ (middle), and with a barotropic EOS using $\rho_{\mathrm{ad}}=2.3 \times 10^{-13} \mathrm{~g} \mathrm{~cm}^{-3}$ and $\gamma=5 / 3($ right). Velocity vectors are overplotted on the density maps 